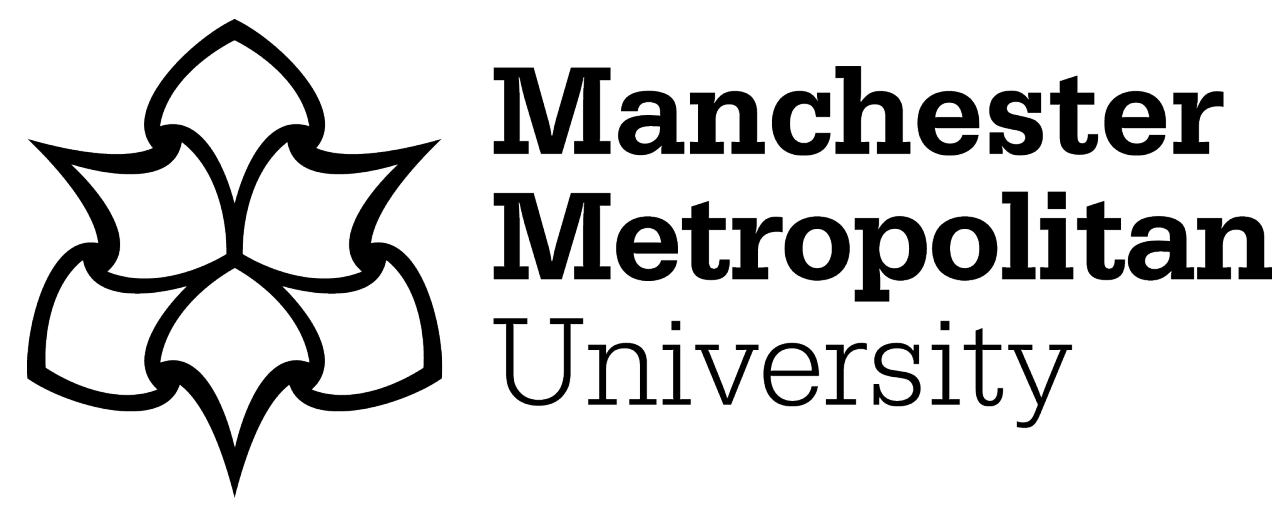

Hernández-Ibáñez, N, Sanjuán, I, Montiel, M, Foster, CW, Banks, CE and Iniesta, J (2016) L-Cysteine determination in embryo cell culture media using Co (II)-phthalocyanine modified disposable screen-printed electrodes. Journal of Electroanalytical Chemistry, 780. pp. 303-310. ISSN 1572-6657

Downloaded from: https://e-space.mmu.ac.uk/617894/

Version: Accepted Version

Publisher: Elsevier

DOI: https://doi.org/10.1016/j.jelechem.2016.09.028

Usage rights: Creative Commons: Attribution-Noncommercial-No Derivative Works 4.0

Please cite the published version 


\title{
L-Cysteine Determination in Embryo Cell Culture Media using Co (II)-phthalocyanine Nanoparticle Modified Disposable Screen-printed Electrodes
}

\author{
Naiara Hernández-Ibáñez ${ }^{a}$, Ignacio Sanjuán ${ }^{a}$, Miguel Ángel Montiel ${ }^{a}$, \\ Christopher W. Fosterc, Craig E. Banks ${ }^{c}$, and Jesús Iniesta ${ }^{a, b *}$ \\ a Institute of Electrochemistry, University of Alicante, 03690, San Vicente del Raspeig, \\ Alicante, Spain \\ ${ }^{\mathrm{b}}$ Physical Chemistry Department, University of Alicante, 03690, San Vicente del Raspeig, \\ Alicante, Spain \\ ${ }^{\mathrm{c}}$ Faculty of Science and Engineering, Manchester Metropolitan University, Chester Street, \\ Manchester M1 5GD UK
}

Submitted to: Journal of electroanalytical chemistry

*Corresponding author: e-mail: jesus.iniesta@ua.es

Tel: +34965909850

Fax: +34965903537 


\begin{abstract}
Thiol-containing compounds such as L-cysteine have been demonstrated to play an important role in metabolism and cellular growth, acting as powerful antioxidants. Consequently, their analytical determination in biological media has received a considerable amount of attention. In this work, an electrochemical sensor for the accurate electroanalytical determination of L-cysteine is proposed, based upon a Co(II)phthalocyanine nanoparticle bulk modified disposable screen-printed graphite electrode (CoPc-SPE). This CoPc mediator has previously been introduced through its incorporation within the bulk of the ink formulation, avoiding the drop-casting method, which has been shown to give a low reproducibility. Cyclic (CV) and Square Wave (SWV) voltammetry experiments have demonstrated an excellent electrocatalytic activity towards the electrochemical oxidation of L-cysteine using CoPc-SPEs within optimum neutral or basic $\mathrm{pH}$. Moreover, the SWV response of L-cysteine is found to exhibit a linear range of $2.6-200 \mu \mathrm{M}$, with a low limit of detection of $4 \mu \mathrm{M}(\mathrm{S} / \mathrm{N}=3)$ and a sensitivity of $0.750 \mu \mathrm{A} \mathrm{cm}^{-2} \mu \mathrm{M}^{-1}$. The CoPc-SPE platforms also display useful reproducibility and the effect of inherent interferences such as amino acids have been evaluated. Finally, the applicability of the L-cysteine electrochemical sensor based upon CoPc-SPEs has been successfully employed for the first time for the assessment of Lcysteine in a complex embryo cell culture medium.
\end{abstract}

Keywords: thiol-containing compounds, L-cysteine, cobalt phthalocyanine, screenprinted electrodes, embryo cell culture media, oxidative stress. 


\section{Introduction}

Low-molecular mass thiols, such as L-cysteine (L-CySH), homocysteine (HCySH) and glutathione (GSH) have been demonstrated to play an important role in cellular growth and metabolism [1]. Specifically, L-CySH is one of the non-essential amino acids, which presents a crucial role in regulating the biological activity of proteins [2] and protecting cells against free radicals (in the cellular antioxidant defense system) [3]. L$\mathrm{CySH}$ deficiency has been related to some pathologies as syndrome of slow growth in children, liver damage, edema, muscle loss, skin lesion and neurodegenerative diseases, among others $[1,4-7]$.

Addition of non-essential amino acids, e.g., L-CySH into an embryo cell culture media increases cell numbers at the blastocyst stage [8]. The importance of this addition has been proven by some groups [9-11] reporting that L-CySH stimulates the synthesis of GSH and improves embryo production. Rahim et al. reported that L-CySH addition in in vitro bovine oocyte maturation enhances cell proliferation [9]. Furthermore, its deficiency in the medium may result in GSH synthesis failure and consequently detrimental outcomes during embryo development [12]. Embryo culture media composition can be very variable between trademarks and between different batches within the same registered products, having L-CySH concentrations quite different among them. In other cases, embryo culture media do not contain L-CySH within their composition and it may be beneficial for its addition. In consideration of the aforementioned reasons the development of a facile, rapid and cost effective method for the L-CySH determination within embryo cell culture media and other biological samples for clinical applications is significant [2]. A vast number of analytical methods have been employed with this purpose, including both high performance liquid chromatography and capillary electrophoresis coupled to fluorescence [13, 14], UV-Vis spectroscopy $[15,16]$, mass spectrometry $[17,18]$ and electrochemical detectors $[19$, 20], as well as methodologies based on fluorescence microscopy [21] and chemiluminescence [7,22]. Nonetheless, although those analytical methods are sensitive, selective and accurate, they are time-consuming, require sample preparation and handling, and are unfeasible for routine analysis [5, 6, 23]. 
The use of an electrochemical sensor may provide a suitable approach in terms of a more economic and sensitive method for L-CySH determination with easy miniaturization, low limit of detection (LoD) and in-situ analyses. Nevertheless, it is worth noting that these methods do lack high/good reproducibility compared to chromatographic methods coupled to other analytical techniques.[24]. Within the field of electroanalysis, previous studies describe that bare carbonaceous materials and conventional metallic electrodes ( $\mathrm{Au}, \mathrm{Pt})$ usually exhibit high overpotentials for the electrooxidation of L-CySH due mainly to a sluggish electron transfer kinetic and, consequently, they exhibit short linear range concentration and low selectivity for LCySH determination [25-27]. To overcome these problems, various chemically modified electrodes (CMEs) and biosensors with electrocatalytic properties have been developed and applied for a superior determination of L-CySH [1-6, 28-34]. In this sense, different types of materials have been employed to modify electrodes surface, e.g. carbon based nanomaterials $[3,4,29]$, metal and metal oxide nanoparticles $[5,6$, 27, 30], graphene oxides [6], nanostructures of different compounds [31, 34, 35], conductive polymers [31, 33, 34] and metallophthalocyanines [1]. More particularly, metallophthalocyanines (MPc, where $\mathrm{M}$ : $\mathrm{Co}, \mathrm{Cu}, \mathrm{Zn}, \mathrm{Fe}$ ) exhibit excellent electronic properties, and potential applications in electrocatalysis towards sulfhydryl compounds electrooxidation [2, 32, 36-38]. MPc-modified electrodes have been employed by many research groups usually through drop-casting, physical adsorption or electropolymerisation of the MPc moiety on a bare electrode surface [2, 32, 39-41], involving laborious synthesis and a lack of stability and reproducibility. As an alternative, the use of bulk-CoPc screen-printed electrodes (CoPc-SPEs) allows the mass production of reproducible electrodes since the $\mathrm{CoPc}$ is incorporated into the ink used to fabricate them. CoPc-SPE disposable platforms have been proved to exhibit excellent electrocatalytic properties towards the electrooxidation of compounds such as ascorbic acid, citric acid and hydrazine with high reproducibility in the electrochemical measurements compared with drop-casted SPEs [42-44]. The use of SPEs has also the big advantage of being economic, providing more reproducibility and repeatability, requiring neither previous polishing nor electrochemical conditioning. To the best of our knowledge, there are very few works which determine L-CySH by SPEs [45-47] and they do not take advantage of MPc electrocatalytic properties through this CoPcSPE platform. 
In this paper, the electrochemical behaviour of L-CySH in a phosphate buffer solution (PBS) $\mathrm{pH} 7.0$ has been performed by cyclic voltammetry (CV) and square wave voltammetry (SWV) utilisng CoPc-SPE platforms in order to explore its electrocatalytic properties towards the electrooxidation of L-CySH and its further feasibility for the determination of complex biological samples. L-CySH electrochemical behaviour has been also examined as a function of $\mathrm{pH}$, analytical concentration, and inherent interferences present within an embryo cell culture medium. Moreover, L-CySH determination has been validated using a synthetic complex medium with a well-known L-CySH concentration and then within embryonic cell culture media commonly employed in in vitro human reproduction using disposable CoPc-SPE platforms.

\section{Material and methods}

\subsection{Reagents and chemicals.}

Amino acids used in this work were purchased from Sigma Aldrich at the highest analytical grade available and were used without further purification. Solutions were prepared using ultrapure water with a resistivity not less than $18.2 \mathrm{M} \Omega \cdot \mathrm{cm}$. Unless otherwise stated, $0.1 \mathrm{M}$ phosphate buffer solution (PBS) were prepared within $\mathrm{pH}$ range between 5.0 and 9.0 by using both $\mathrm{Na}_{2} \mathrm{HPO}_{4}$ and $\mathrm{NaH}_{2} \mathrm{PO}_{4}$ in a proper mole ratio (both from Merck, $99 \%$ ). Phosphate buffer solutions at more extreme $\mathrm{pH}$ values were made up setting pH values with phosphoric acid (J. T. Backer, 99-100 \%) and concentrated $\mathrm{NaOH}$ solution (Scharlau Chemie S.A., reagent grade). $\mathrm{pH}$ measurements were carried out with a Crison Micro pH 2000 pH-meter.

A free Human Serum Albumin protein (HSA) G2 type cell culture medium was purchased from Vitrolife. Though the qualitative and quantitative composition of the cell culture medium remains under confidential, the prospect information of our G2 medium describes the presence of L-cystine instead of L-CySH. G2 medium was stored in the refrigerator at $4{ }^{\circ} \mathrm{C}$. L-CySH standards were always freshly prepared using ultrapure water and then also stored at $4{ }^{\circ} \mathrm{C}$ before the electrochemical measurements.

\subsection{Electrochemical Configuration.}

Electrochemical sensor consisted of a three-electrode system using cobalt (II) phthalocyanine screen-printed electrodes (CoPc-SPE) and screen-printed graphite 
electrodes (SPEs) were also fabricated for comparative studies. Both CoPc-SPE and bare SPE electrochemical platforms were fabricated in-house with appropriate stencil designs to achieve a $3 \mathrm{~mm}$ diameter working electrode $\left(0.071 \mathrm{~cm}^{2}\right.$ projected area), using a microDEK 1760RS screen-printing machine (DEK, Weymouth, UK). In the case of SPE fabrication, carbon graphite ink formulation (Product Code: C2000802P2; Gwent Electronic Materials Ltd., Pontypool, UK) was first printed onto the polyester $(250 \mu \mathrm{m}$ thickness, Autostat TM, Oxford, UK). This layer was cured in a fan oven at $60{ }^{\circ} \mathrm{C}$ for 30 min. Next a silver/silver chloride (40:60) reference electrode was applied by screenprinting $\mathrm{Ag} / \mathrm{AgCl}$ paste (Product Code: C2040308P2; Gwent Electronic Materials Ltd, UK) onto the plastic substrate. This layer was once more cured in a fan oven at $60{ }^{\circ} \mathrm{C}$ for 30 minutes. Finally, a dielectric paste (Product Code: D2070423D5; Gwent Electronic Materials Ltd.) was then printed onto the polyester substrate to cover the connections. After curing at $60{ }^{\circ} \mathrm{C}$ for 30 minutes the screen-printed electrodes were ready to be used. Regarding CoPc-SPE fabrication, a carbon-graphite ink formulation together with the mediator CoPc (Product code: C2030408P3; Gwent Electronic Materials Ltd.) was used. The same printing procedure described above was also applied to manufacture the CoPc-SPE. Both CoPc-SPE and SPE platforms has been extensively physico-chemical characterized in previous works by Foster et al. [42, 44]. The electrochemical surface areas of the bare SPE platforms were determined using the Randles-Ševčík equation for a quasi-reversible and fast electron transfer process [48]. To determine this, the outer-sphere redox probe hexaamineruthenium (III)/(II) chloride (from Sigma Aldrich) was dissolved in $0.1 \mathrm{M}$ phosphate buffer solution ( $\mathrm{pH} 7.0)$ to reach a final concentration of $1.0 \mathrm{mM}$. Such results provided an electroactive area of $0.052 \mathrm{~cm}^{2}$ for the SPE platform with a coefficient of variation of $6.22 \%(N=6)$. In the case of the electrode active area of CoPc-SPE, the outer-sphere redox probe was 1.0 $\mathrm{mM}$ potassium ferro/ferricyanide (from Sigma Aldrich) in 1.0 M KCl solution. The electrochemical surface area resulted to be $0.055 \mathrm{~cm}^{2}$ for the CoPc electrode, with a coefficient of variation of $3 \%(N=5)$.

\subsection{Electrochemical measurements}

Cyclic voltammetry (CV) and square wave voltammetry (SWV) measurements were carried out using an Autolab PGSTAT 30 (Eco Chemie, The Netherlands) potentiostat/galvanostat and controlled by Autolab GPES software version 4.9 for Windows XP. All electrochemical experiments were carried out under aerated 
conditions and at $22 \pm 2{ }^{\circ} \mathrm{C}$. Optimized SWV parameters used in all experiments were the following: modulation amplitude, $50 \mathrm{mV}$; modulation frequency, $10 \mathrm{~Hz}$; modulation step, $5 \mathrm{mV}$. All potentials are referred to an $\mathrm{AgCl} / \mathrm{Ag}$ pseudo-reference electrode. Prior to all the electrochemical measurements, a conditioning pre-treatment of the CoPc-SPE working electrode was performed, in which the electrode was submitted to a CV between 0 and $1.0 \mathrm{~V}$ at a scan rate of $50 \mathrm{mV} / \mathrm{s}$ in $0.1 \mathrm{M}$ PBS at $\mathrm{pH} 7.0$ to remove any possible surface contimination. Thereafter, both the CoPc-SPE and SPE platforms were rinsed with the same buffer solution. Generally, $50 \mu \mathrm{L}$ of standards or real samples were placed onto the screen-printed electrode before starting CV or SWV measurements. Unless otherwise stated, CoPc-SPE or SPE were discarded after a single use.

Equations for calibration linear plots were obtained by the least squares method with the help of the spreadsheet application for calculus, Microsoft EXCEL 2010. Confidence intervals of the slope and the intercept were obtained using the statistical value " $t$ student"' (for N-2 freedom degrees, where $\mathrm{N}$ is the number of standards solutions used) for a confidence level of $95 \%$. Limit of detection (LoD) and quantification (LoQ) were calculated as three and ten times the noise level. Sensitivities were expressed in $\mu \mathrm{A} \mathrm{cm}-2$ $\mu \mathrm{M}^{-1}$ using the electrochemcal surface area obtained from the electrochemical procedure used before.

\subsection{Analysis of the cell culture embryonic samples}

The proposed procedure was applied to the feasibility of analysing L-CySH in cell culture embryonic media. G2 type cell culture medium which does not contain neither L-CySH nor HSA protein was chosen as a complex biological fluid. Known amount of L-CySH were spiked into the G2 cell culture embryonic medium and further diluted ten times by using 0.1 M PBS buffer solution $\mathrm{pH}$ 7.0. No further pre-treatment was needed before the electrochemical measurement using the CoPc electrochemical disposable sensor. Added and found L-CySH concentrations in cell culture media were then compared along the apparent, recoveries. 


\section{Results and discussion}

\section{1. $C V$ and $S W V$ behaviour of L-CySH at CoPc-SPE.}

Figure 1 depicts the electrochemical response of L-CySH obtained using CoPc-SPEs, which is compared to a bare SPE via CV and SWV in a $50 \mu \mathrm{M} \mathrm{L-CySH}$ solution within a $0.1 \mathrm{M}$ phosphate buffer solution (PBS) $\mathrm{pH}$ 7.0. The $\mathrm{CV}$ as shown in Figure 1A exhibits two main irreversible anodic peaks at $+0.10 \mathrm{~V}$ and at $+0.59 \mathrm{~V}$ at the CoPc-SPE, whereas only one anodic peak at $+0.53 \mathrm{~V}$ is visible when using the bare SPE. Similar results are found when SWV is applied, as shown in Figure 1B where two anodic peaks appear at $0 \mathrm{~V}$ and $+0.49 \mathrm{~V}$ for the CoPc-SPE and again only one anodic peak located at $+0.41 \mathrm{~V}$ for the bare SPE.

\section{HERE FIGURE 1}

Fig.1. (A) CV responses of $50 \mu \mathrm{M}$ L-CySH in $0.1 \mathrm{M}$ PBS pH 7.0 using CoPc-SPE (red line) and bare SPE (black line). Scan rate: $50 \mathrm{mV} \mathrm{s}^{-1}$. (B) SWV responses of $50 \mu \mathrm{M} \mathrm{L}-$ CySH in 0.1 M PBS pH 7.0 using CoPc-SPE (red line) and bare SPE (black line). Background responses are shown as dashed lines.

The voltammograms recorded in Figure $1 \mathrm{~B}$ obtained for the SPE and the SPE containing CoPc electrodes suggest that the oxidation mechanisms are distinct at both electrodes, and the difference in peak potential of about $80 \mathrm{mV}$ for the second oxidative wave occurring at both electrodes can be attributed to slight differences in the pseudoreference electrode or simply to different surface chemistry of the carbonaceous electrode ascribed to electrode preparation.

As clearly observed, the L-CySH electrochemical response differs noticeably when CoPc is introduced into the ink of the SPE, revealing an electrocatalysis effect due to the CoPc presence $[2,32,49]$. This fact is of great interest since the electrochemical oxidation of L-CySH at CoPc-SPEs exhibits a low overpotential of the oxidation peak, which is important in terms of selectivity, potentially discarding/overcoming many inherent interference that are likely to be present within real samples. Accordingly, we explore the first anodic peak, $\sim 0 \mathrm{~V}$, of L-CySH at CoPc-SPE by CV and SWV with a potential range recorded between $-0.2 \mathrm{~V}$ and $+0.3 \mathrm{~V}$, as depicted in Figure 2. The 
reusability of these CoPc-SPE platforms was studied upon five consecutive CV or SWV experiments within a $50 \mu \mathrm{M}$ L-CySH in a $0.1 \mathrm{M}$ PBS pH 7.0, washing the electrode thoroughly with ultrapure water after each electrochemical experiment. It is found that the anodic response remains stable and reproducible; therefore, the catalytic surface based CoPc redox mediator remains active. Nevertheless, consecutive CV or SWV measurements without washing the electrode resulted in a loss of peak current and therefore fouling phenomenon is taking place associated with reaction products formation. Furthermore, both CV and SWV experiments performed in Figure 2 show well-defined anodic peaks with stable peak potentials at $+0.03 \mathrm{~V}$ and $-0.04 \mathrm{~V}$, respectively.

\section{HERE FIGURE 2}

Fig. 2. CV (A) and SWV (B) electrochemical oxidation of $50 \mu \mathrm{M}$ L-CySH in $0.1 \mathrm{M}$ PBS pH 7.0 (solid lines) using CoPc-SPE. Background responses are shown as dashed lines. Scan rate: $50 \mathrm{mV} \mathrm{s}^{-1}$. Five consecutive voltammograms are shown following gentle washing of the CoPc-SPE between electrochemical measurements.

By comparing the electrochemical response of backgrounds in Figures 1A and 2A, the behavior are completely different. The small reduction peak observed in Figure 2A on the negative direction does not appear in Figure 1A, which can be attributed to the use of a distinct potential window. Nevertheless, that small anodic peak does not interfere in the measurement of the anodic peak current obtained in the presence of L-CySH.

A high reproducibility of the electrochemical response of a $50 \mu \mathrm{M} \mathrm{L-CySH}$ solution in $0.1 \mathrm{M}$ PBS at $\mathrm{pH} 7.0$ was also demonstrated at different CoPc-SPEs providing a coefficient of variation $(\mathrm{CoV})$ of $3 \%$ when five different electrodes were used $(N=5)$, as shown in Figure 3.

\section{HERE FIGURE 3}

Fig. 3. SWVs of a $50 \mu \mathrm{M}$ L-CySH solution in $0.1 \mathrm{M}$ PBS pH 7.0 at different CoPcSPEs. 
Furthermore, the electrochemical behaviour of L-CySH was explored in terms of potential and current dependence with the scan rate through $\mathrm{CV}$ measurements. Figure 4 depicts the CV response of a $50 \mu \mathrm{M}$ L-CySH solution in $0.1 \mathrm{M}$ PBS pH 7.0 with scan rate, and such results reveal that peak current $\left(\mathrm{I}_{\mathrm{p}}\right)$ behaves linearly with square root of the scan rate. Moreover, peak potential and peak current logarithm with the logarithm of scan rate exhibit a linear dependence. In addition, the plot $\log _{10}\left(\mathrm{I}_{\mathrm{p}} / \mu \mathrm{A}\right)$ versus $\log _{10}[\mathrm{v} /$ $\left(\mathrm{mV} \mathrm{s}^{-1}\right)$ gives rise to a linear dependence $\left(R^{2}=0.998\right)$ with a slope of 0.4988 , which is almost the same that the theoretical value of 0.5 for a diffusion controlled process [50]. Moreover, the dependence of peak potential with scan rate denotes L-CySH electrooxidation is an irreversible process.

\section{HERE FIGURE 4}

Fig. 4. Typical CV responses of $50 \mu \mathrm{M}$ L-CySH in $0.1 \mathrm{M}$ PBS pH 7.0 as a function of voltammetric scan rate. Scan rates: $10,20,50,75,100,200,500 \mathrm{mV} \mathrm{s}^{-1}$. Inset figure shows the plot of $\log 10 \mathrm{I}_{\mathrm{p}}$ versus Log v. First scan utilised.

\subsection{Effect of $p H$ on the L-CySH electrochemical response at CoPc-SPE.}

Figure $5 \mathrm{~A}$ depicts the $\mathrm{pH}$ dependence upon the electrochemical oxidation of L-CySH which has been investigated towards a $50 \mu \mathrm{M}$ L-CySH solution in $0.1 \mathrm{M}$ PBS over a $\mathrm{pH}$ range of 4.64 to 10.01 . Results indicate that peak potentials shift to lower positive values with $\mathrm{pH}$. A linear $\mathrm{pH}$-dependence with peak potential gives rise to a slope of 105 $\mathrm{mV} \mathrm{pH}^{-1}$, as observed in Figure. 4B. Strikingly, this slope does not correspond to the Nernstian value of $59 \mathrm{mV}$ per $\mathrm{pH}$ corresponding to $2 \mathrm{H}^{+} / 2 \mathrm{e}^{-}$process, which is the proposed and accepted by many authors for the L-CySH electrooxidation to L-cystine upon carbon based materials and electrodes modified with electrocatalysts such as CoPc, conductive polymers or Au nanoparticles [1, 2, 46, 51], according to reaction $(1)$,

$$
2 L-C y S H \leftrightarrow C y S S C y+2 H^{+}+2 e^{-}
$$


A value of $105 \mathrm{mV} \mathrm{pH}^{-1}$, as obtained from Figure 5B, could be attributed to the complexity of the electron transfer reactions upon the complex mediator $\mathrm{CoPc}$ and/or the participation of different ionic species as a result of acid-base L-CySH equilibriums, whose $\mathrm{pK}_{\mathrm{a}}$ are 1.71, 8.36, and 10.77 [51]. In this regard, it should be noted that within the studied range of $\mathrm{pH}$ a change in the slope of the $\mathrm{E}_{\mathrm{p}} \mathrm{vs} \mathrm{pH}$ plot can be detected at $\mathrm{pH}$ 8.6 (highlighted with arrow in Figure 5B). This deviation from linearity correlates well with the $\mathrm{pK}_{\mathrm{a} 2}$ of L-CySH, as already reported by the literature $[2,51]$.

\section{HERE FIGURE 5}

Fig. 5. (A) SWV responses of a $50 \mu \mathrm{M}$ L-CySH using CoPc-SPE as a function of $\mathrm{pH}$. $0.1 \mathrm{M}$ PBS adjusted at $\mathrm{pH}$ values between 4.64 and 10.01. (B) Shows a plot of peak potential versus $\mathrm{pH}$. Scan rate: $50 \mathrm{mV} \mathrm{s}^{-1}$; First scan recorded.

At acidic pHs, the electrochemical behaviour of L-CySH is different compared to basic and neutral pHs as we can observe in Figure 6A, where the appearance of an anodic peak at $+0.49 \mathrm{~V}$ at $\mathrm{pH} 3.0$ seems to indicate that the molecule reacts through a different mechanism. The single anodic peak occurring between the potential range of -0.2 and $+0.2 \mathrm{~V}$ disappears whereas the additional anodic peak at approximately $+0.5 \mathrm{~V}$ increases upon the forward scan. Interestingly, during the reverse scan, L-CySH is yet again catalytically oxidised at the same potential. This behaviour has been already observed by pioneering work performed by Halbert and Baldwin [38] for the electrooxidation of different sulfhydryl compounds at $\mathrm{CoPc}$ modified carbon paste electrodes, and also for the electrocatalytic oxidation of other biomolecules e.g. citric acid, hydrogen peroxide and hydrazine, as stated in recent works by Foster et al. [42, 44], with still uncertain explanation for this behaviour.

Within the experimental errors, an almost constant current for the anodic oxidation of L$\mathrm{CySH}$ is observed upon an increase within the solution $\mathrm{pH}$, as shown in Figure 6B. It is clear that $\mathrm{pH}$ solution influences on the L-CySH electrooxidation, due to the L-CySH molecule ionization. At pHs higher than 8.4, L-CySH entity is mostly deprotonated (in the form $\mathrm{HCys}^{-}$), therefore it reacts faster upon the CoPc-SPE since both a deprotonation step is not involved in the mechanistic reaction [52-54] and the thiolate 
group $\left(-\mathrm{S}^{-}\right)$is actually the electroactive moiety, achieving low oxidation potential and slightly high currents [51]. In the case of $\mathrm{pHs}$ between 4 and 8 , the main ionization form of L-CySH corresponds to the zwitterion state $\left(\mathrm{H}_{2} \mathrm{Cys}\right)$ which reacts slower than the deprotonated form $\left(\mathrm{HCys}^{-}\right)$upon the CoPc-SPE since the thiol deprotonation must be carried out previously. Similar results were achieved by Zhou et al [55], indicating a different reaction mechanisms for the electrochemical oxidation of $\mathrm{L}-\mathrm{CySH}$ according to solution $\mathrm{pH}$. It seems that changes in composition of $\mathrm{L}-\mathrm{CySH}$ ionic forms with $\mathrm{pH}$ may explain the $\mathrm{pH}$-dependent electrochemical behaviour.

\section{HERE FIGURE 6}

Fig. 6. (A) Cyclic voltammograms for a $50 \mu \mathrm{M}$ L-CySH solution in $0.1 \mathrm{M}$ PBS at pHs between 3 and 7 using CoPc-SPE. Scan rate: $50 \mathrm{mV} \mathrm{s}^{-1}$. First scan recorded. (B) pHdependence of the voltammetric peak current obtained using CV responses in $50 \mu \mathrm{M} \mathrm{L}$ CySH recorded within 0.1 M PBS at pHs between 4.64 and 10.01.

We next turned out the L-CySH electrooxidation behaviour from a mechanistic and kinetic point of view. Firstly, from the peak potential dependence with logarithm of the scan rate the kinetic parameter $\left(\alpha \cdot n_{A}\right)$ was calculated by using [48]:

$E_{p}=$ cte. $+\frac{1}{2} \cdot \frac{R T}{\left(\alpha \cdot n_{A}\right) \cdot F} \cdot 2.3 \cdot \log (v) ; b=\frac{R T}{\left(\alpha \cdot n_{A}\right) \cdot F} \cdot 2.3$

where $\mathrm{n}_{\mathrm{A}}$ is the number of electrons exchanged through the rate-determining step, $\alpha$ is the electronic transfer coefficient, $\mathrm{F}$ is the Faraday constant $\left(96,485 \mathrm{C} / \mathrm{mol} \mathrm{e}^{-}\right)$and $\mathrm{R}$ is the molar gas constant $\left(8.31 \mathrm{~J} \cdot \mathrm{K}^{-1} \cdot \mathrm{mol}^{-1}\right)$. Therefore, from the slope value of the plot $\mathrm{E}_{\mathrm{p}}$ versus $\log \mathrm{V}\left(0.0549 \mathrm{~V} \mathrm{\operatorname {dec } ^ { - 1 }}\right)$, the value of $\left(\alpha \cdot \mathrm{n}_{\mathrm{A}}\right)$ can be estimated as 0.54 . Additionally, Tafel slope $(b)$ can be determined as $110 \mathrm{mV}$ (close to the theoretical value of $118 \mathrm{mV}$ ) [48]. Assuming that $\mathrm{n}_{\mathrm{A}}$ is 1 , we can assert $\alpha$ with a value of 0.54 . Secondly, by knowing the value of $\left(\alpha \cdot \mathrm{n}_{\mathrm{A}}\right)$ now, we next proceeded to estimate the number of electrons exchanged through the whole electrochemical process from the plot peak current versus the square root of the scan rate, according to the $\mathrm{CV}$ experiments. For an irreversible process, that dependence follows the equation (3) [48]. 
$I_{p}=\left(2.99 \cdot 10^{5}\right) \cdot\left(n_{A} \cdot \alpha\right)^{1 / 2} \cdot n \cdot D^{1 / 2} \cdot A \cdot C \cdot v^{1 / 2}$

where $\mathrm{n}$ is the number of electrons involved in the mechanism, $\mathrm{A}$ is the electrode area $\left(\mathrm{CoPc}-\mathrm{SPE}\right.$ area $\left.=0.055 \mathrm{~cm}^{2}\right), \mathrm{D}$ is the L-CySH diffusion coefficient (assumed to be: 3 . $10^{-5} \mathrm{~cm}^{2} \mathrm{~s}^{-1}$ [56]) and $\mathrm{C}$ is the concentration of the electroactive species (L-CySH, 50 . $\left.10^{-9} \mathrm{~mol} \mathrm{~cm}{ }^{-3}\right)$. A slope of $7.59 \cdot 10^{-6} \mathrm{~A}\left[\mathrm{~V} \mathrm{~s}^{-1}\right]^{-1 / 2}$ was obtained, thereby the number of electrons involved in the electrooxidation mechanism calculated from (3) was 2.3, which can be approximated to 2 . Such value corresponds with the proposal mechanism in (1) and is in agreement with the literature [52-54]. Besides, plot of peak current logarithm as a function of logarithm of L-CySH concentration exhibits a linear trend with a slope value of 1.024 . This indicates that the electrochemical oxidation is a first order reaction and thus the rate-determining step corresponds to the thiol radical formation.

In summary, our results reveal that neutral and basic conditions are more adequate for L-CySH determination using the CoPc-SPE in terms of achieving a low overpotential and therefore minimizing the effect of inherent interferences, which can be oxidised at more positive potentials. For that reason, a $\mathrm{pH}$ value of 7.0 was chosen as the working $\mathrm{pH}$ for the electroanalytical determination of $\mathrm{L}-\mathrm{CySH}$ in an embryo cell culture medium.

\subsection{Analytical figures of merit}

Hereafter, for the electroanalytical determination of $\mathrm{L}-\mathrm{CySH}$, its electrooxidation was followed by SWV in order to reach a higher sensitivity and lower LoD when compared with CV measurements [57]. Electroanalytical quantification of L-CySH was accomplished in 0.1 M PBS pH 7.0, as depicted in Figure 7, with concentrations varying between 2.6 and $200 \mu \mathrm{M}$. A linear plot of anodic peak current versus L-CySH concentration was found with correlation: $\mathrm{I}_{\mathrm{p}} / \mu \mathrm{A}=(0.043 \pm 0.002)[\mathrm{L}-\mathrm{CySH} / \mu \mathrm{M}]+(0.1$ $\pm 0.2)\left(R^{2}=0.997, N=2\right)$. The corresponding value for the LoD was found to be 3.8 $\mu \mathrm{M}$ whereas the LoQ was $6.0 \mu \mathrm{M}$, based on three and ten times the noise level, respectively. 


\section{HERE FIGURE 7}

Fig. 7. SWV responses of L-CySH standard solutions with increasing concentrations $(2.6,5,10,25,50,100,150,200 \mu \mathrm{M})$ made into a 0.1 M PBS pH 7.0 using CoPc-SPE. Inset Figure shows the resulting calibration plot of peak current versus L-CySH concentration.

The effect of the presence of the electroactive species within a solution, which can interfere with the electroanalytical L-CySH determination, is of great importance for the validation of an electrochemical sensor. Most of sulphur-containing compounds such as L-cystine, L-cysteic acid and DL-methionine (Met) can be present in the formulation of a biological sample or simply some of them can be yielded during the development of embryonic cells. After the analysis of the electrochemical response of each compound, as shown in Figure 8, we can notice that Met exhibits a significant electrochemical anodic wave at $+0.78 \mathrm{~V}$ using SWV.

\section{HERE FIGURE 8}

Fig. 8. SWV responses for the electrooxidation of $50 \mu \mathrm{M}$ DL-Met (pink trace), $100 \mu \mathrm{M}$ L-Cystine (blue line) and $100 \mu \mathrm{M}$ L-Cysteic acid (red line) solutions using CoPc-SPE electrochemical sensors. SWV response of $0.1 \mathrm{M}$ PBS pH 7.0 is highlighted in a black dashed trace.

Met is another amino acid usually added into cell culture media, therefore we next analyse the electrochemical response of $\mathrm{L}-\mathrm{CySH}+\mathrm{Met}$ mixtures in a 1 to 1 concentration ratio via SWV in order to prove whether Met affects the L-CySH response at CoPc-SPE platforms. Figure 9 depicts that in the presence of Met, L-CySH determination is feasible since there is a linear relationship between peak current $(\sim 0 \mathrm{~V})$ and L-CySH concentration but with a reduction in sensitivity of $30 \%$ compared to that obtained in Figure 6. By contrast, the L-CySH anodic peak remains unaltered by the presence of Met, whereas Met anodic wave shifts $100 \mathrm{mV}$ to more positive potentials compared to that obtained in Fig. ESI-5 upon CoPc-SPEs.

\section{HERE FIGURE 9}


Fig. 9. SWV responses of L-CySH + DL-Met mixtures in 1:1 concentrations ratio (both with $3,6,13,25,50,50,75$, and $100 \mu \mathrm{M})$. Inset figure shows the calibration plot of peak current at $\sim 0 \mathrm{~V}$ versus L-CySH concentration. Background response is recorded in a black dashed line using CoPc-SPE.

Despite the examination of L-cystine, L-cysteic acid and Met as interferents for the L$\mathrm{CySH}$ determination, the interference effect was further investigated in the presence of high amounts of different amino acids that are present in an embryo cell culture medium. Thus, L-CySH standards were prepared under the simultaneous presence of $300 \mu \mathrm{M}$ of L-tryptophan, L-tyrosine, L-serine, L-asparagine, L-glutamine, L-glutamic acid, L-alanine, L-proline, L-methionine, L-aspartic acid, L-histidine and Lphenylalanine, in a $0.1 \mathrm{M}$ PBS $\mathrm{pH} 7.0$, to explore the L-CySH determination in a complex matrix by SWV. The correlation between peak current at near $0 \mathrm{~V}$ and L$\mathrm{CySH}$ concentration was evaluated by SWV measurements and then a calibration linear plot was obtained, as shown in Figure 10.

\section{HERE FIGURE 10}

Fig. 10. SWV responses for the electrooxidation of $50 \mu \mathrm{M}$ DL-Met (pink trace), 100 $\mu \mathrm{M}$ L-Cystine (blue line) and $100 \mu \mathrm{M}$ L-Cysteic acid (red line) solutions using CoPcSPE electrochemical sensors. SWV response of $0.1 \mathrm{M}$ PBS pH 7.0 is highlighted in a black dashed trace.

Equation of the linear regression was $\mathrm{I}_{\mathrm{p}} / \mu \mathrm{A}=0.0054[\mathrm{~L}-\mathrm{CySH} / \mu \mathrm{M}]-0.369$, with a sensitivity about ten times lower than that obtained for a calibration curve of L-CySH in the absence of interferences (see inset Figure 7). Accordingly, applicability of our electrochemical sensor within the presence of interferents was explored via the recovery method. Given amounts of L-CySH were spiked into the containing interference sample (with $114 \mu \mathrm{M} \mathrm{L-CySH}$ ) and the electrochemical response of the resulting sample was recorded. Subsequently, by using the equation of the line, L-CySH concentration was determined for each addition and the recovery was also calculated. Analytical results of the addition standard method were summarized in Table 1. According to these results, there is no significant differences between the added L-CySH concentration and that 
found by our electrochemical sensor in synthetic cell culture media, with recoveries ranging between $95 \%$ and $101 \%$, thereby there is no significant matrix effect and therefore L-CySH determination in the presence of a high amount of amino acids [58], could be feasible using CoPc-SPEs in real cell culture media.

Table 1. L-CySH determination in spiked synthetic cell culture medium using CoPc-SPEs.

\begin{tabular}{ccc}
\hline $\begin{array}{c}\text { Added concentration } \\
(\boldsymbol{\mu M})\end{array}$ & $\begin{array}{c}\text { Found concentration } \pm \text { SD } \\
(\boldsymbol{\mu M})\end{array}$ & Apparent recoveries (\%) \\
\hline $\mathbf{1 4 6}$ & $146 \pm 2$ & 100.6 \\
$\mathbf{1 7 8}$ & $179.8 \pm 1.5$ & 101.0 \\
$\mathbf{2 4 1}$ & $240.0 \pm 1.0$ & 99.9 \\
$\mathbf{3 9 8}$ & $390.6 \pm 0.4$ & 98.1 \\
\hline
\end{tabular}

SD: standard deviation

\subsection{L-CySH determination in a real embryo cell culture medium.}

The electrochemical sensor based on the use of CoPc-SPE platforms was next applied to the determination of L-CySH within a real sample such as embryo cell culture media. This type of media is very complex and, consequently may appear detrimental matrix effect upon the L-CySH determination as well as a high potential electrode surface fouling. To assess the applicability of the CoPc-SPE an embryo cell culture medium (G2 Vitrolife) was selected for the analysis of L-CySH. Given amounts of L-CySH in the millimolar concentration were spiked into the real embryo cell culture medium, obtaining a linear correlation between peak current versus L-CySH concentration, as shown in Figure 11.

\section{HERE FIGURE 11}

Fig. 11. SWV responses for the electrooxidation of L-CySH within a G2 Vitrolife cell culture medium upon different L-CySH concentrations $(2.5,5.0,7.0,9.0,10.0,12.0$ $\mathrm{mM})$ at CoPc-SPE. Inset figure depicts the calibration plot of peak current recorded at 0 $\mathrm{V}$ as a function of L-CySH concentration. 
Unfortunately, in the micromolar concentrations, the electrochemical response of L$\mathrm{CySH}$ was unfeasible under our experimental conditions. This can be attributed to the effect of the matrix on the fouling and/or inactivation of the CoPc catalyst upon the SPE surface. We speculate that the presence of high osmolarity as well as the presence of the biopolymer hyaluronic acid may have detrimental effects on the performance of the CoPc-SPE platform. To overcome this drawback, G2 medium was diluted ten times using 0.1 M PBS pH 7.0 in order to minimize the matrix effect and then given amounts of L- CySH were spiked into the G2 solution. The SWV response of L- CySH electrooxidation together with a linear correlation of peak current versus concentration was obtained at a peak potential of $0 \mathrm{~V}$, as depicted in Figure 12.

\section{HERE FIGURE 12}

Fig. 12. SWV responses resulting from the electrooxidation of L-CySH as a function of L-CySH concentration $(27.0,51.0,80.0,124.0,166.0 \mu \mathrm{M})$ within a G2 Vitrolife cell culture medium using CoPc-SPE. SWV response of the G2 Vitrolife cell culture medium is recorded and shown as the black dashed trace.

The equation of the linear correlation was $\mathrm{I}_{\mathrm{p}} / \mu \mathrm{A}=0.00662[\mathrm{~L}-\mathrm{CySH} / \mu \mathrm{M}]-0.003315$ $\left(R^{2}=0.995\right)$ with a $\mathrm{CoV}$ of $5 \%$ with three different electrodes $(N=3)$. Table 2 summarizes the recoveries of spiked L-CySH concentrations present in G2 samples evaluated by the standard addition method using the regression line. Yet again, as shown in Table 2, the recoveries obtained were quite satisfactory ranging between 97 and $103 \%$, with mostly no significant differences between the added and found L$\mathrm{CySH}$ concentrations, and no relevant matrix effect.

Table 2. L-CySH determination in spiked G2 embryo cell culture medium using CoPc-SPEs.

\begin{tabular}{ccc}
\hline $\begin{array}{c}\text { Added concentration } \\
(\boldsymbol{\mu M})\end{array}$ & $\begin{array}{c}\text { Found concentration } \pm \text { SD } \\
(\boldsymbol{\mu M})\end{array}$ & $\begin{array}{c}\text { Apparent recoveries (\%) } \\
\mathbf{5 0}\end{array}$ \\
$\mathbf{6 5}$ & $48.9 \pm 1.2$ & 97.9 \\
$\mathbf{9 0}$ & $66 \pm 3$ & 102.4 \\
\hline
\end{tabular}

SD: standard deviation. 


\section{Conclusions}

The feasibility of a CoPc-SPE as a sensor for the electroanalytical determination of LCySH within a real embryo cell culture medium has been demonstrated. Voltammetric exploration has revealed that the solution $\mathrm{pH}$ has a strong influence upon the electrochemical response of L-CySH. CoPc-SPE electrochemical sensors have been validated in terms of linearity, sensitivity, LoD, LoQ, repeatability and reproducibility, obtaining a calibration curve over a concentration range of $2.6-200 \mu \mathrm{M}$, with suitable regression $\left(R^{2}=0.997\right)$ and sensitivity of $0.750 \mu \mathrm{A} \mathrm{cm}^{-2} \mu \mathrm{M}^{-1}$. Furthermore, the CoPcSPEs exhibit useful selectivity towards the electrochemical oxidation of L-CySH in the presence of high concentrations of inherent interferences such as a wide handful of amino acids. Finally, the applicability of the successful CoPc-SPE electrochemical sensor has been validated for L-CySH determination within a real embryo cell culture medium. Overall this electrochemical sensor has the potential for real and rapid analytical assessment of L-CySH within a real cell culture media after retrieval of human embryos in assisted reproduction techniques.

\section{Acknowledgement}

J. Iniesta thanks the programme Salvador de Madariaga from the Ministerio de Economía y Competitividad, grant number PRX14/00363. This work has been also financially supported by the MICINN-FEDER (Spain) through the projects CTQ2013-48280-C3-3-R. 


\section{References}

[1] M.N. Abbas, A.A. Saeed, B. Singh, A.A. Radowan, E. Dempsey, A cysteine sensor based on a gold nanoparticle-iron phthalocyanine modified graphite paste electrode, Analytical Methods 7 (2015) 2529-36.

[2] H. Hosseini, M. Mahyari, A. Bagheri, A. Shaabani, A novel bioelectrochemical sensing platform based on covalently attachment of cobalt phthalocyanine to graphene oxide, Biosensors and Bioelectronics 52 (2014) 136-42.

[3] M.A. Khalilzadeh, H. Karimi-Maleh, V.K. Gupta, A Nanostructure Based Electrochemical Sensor for Square Wave Voltammetric Determination of L-Cysteine in the Presence of High Concentration of Folic Acid, Electroanalysis 27 (2015) 1766-73.

[4] A. Benvidi, M.M. Ansaripour, N. Rajabzadeh, H.R. Zare, B.-B.F. Mirjalili, Developing a nanostructure electrochemical sensor for simultaneous determination of cysteine and tryptophan, Analytical Methods 7 (2015) 3920-8.

[5] E. Sharifi, A. Salimi, E. Shams, DNA/nickel oxide nanoparticles/osmium(III)complex modified electrode toward selective oxidation of 1-cysteine and simultaneous detection of 1-cysteine and homocysteine, Bioelectrochemistry 86 (2012) 9-21.

[6] S. Ge, M. Yan, J. Lu, M. Zhang, F. Yu, J. Yu, X. Song, S. Yu, Electrochemical biosensor based on graphene oxide-Au nanoclusters composites for 1-cysteine analysis, Biosensors and Bioelectronics 31 (2012) 49-54.

[7] C. Lau, X. Qin, J. Liang, J. Lu, Determination of cysteine in a pharmaceutical formulation by flow injection analysis with a chemiluminescence detector, Analytica Chimica Acta 514 (2004) 45-9.

[8] E.S. Ginsburg, C. Racowsky, In Vitro Fertilization: A Comprehensive Guide, Springer New York, 2012.

[9] B. Rahim, S. Jalal, N. Yosef, Effect of cysteine supplementation on in vitro maturation of bovine oocyte, African Journal of Biotechnology 10 (2013) 15830-3.

[10] B. Gasparrini, A. De Rosa, L. Attanasio, L. Boccia, R. Di Palo, G. Campanile, L. Zicarelli, Influence of the duration of in vitro maturation and gamete co-incubation on the efficiency of in vitro embryo development in Italian Mediterranean buffalo (Bubalus bubalis), Animal Reproduction Science 105 (2008) 354-64.

[11] L.R. Abeydeera, W.-H. Wang, T.C. Cantley, R.S. Prather, B.N. Day, Glutathione content and embryo development after in vitro fertilisation of pig oocytes matured in the presence of a thiol compound and various concentrations of cysteine, Zygote 7 (1999) 203-10.

[12] J.-i. Sagara, K. Miura, S. Bannai, Cystine Uptake and Glutathione Level in Fetal Brain Cells in Primary Culture and in Suspension, Journal of Neurochemistry 61 (1993) 1667-71.

[13] H. Wang, S.-C. Liang, Z.-M. Zhang, H.-S. Zhang, 3-Iodoacetylaminobenzanthrone as a fluorescent derivatizing reagent for thiols in high-performance liquid chromatography, Analytica Chimica Acta 512 (2004) 281-6.

[14] K. Arlt, S. Brandt, J. Kehr, Amino acid analysis in five pooled single plant cell samples using capillary electrophoresis coupled to laser-induced fluorescence detection, Journal of Chromatography A 926 (2001) 319-25.

[15] A.E. Katrusiak, P.G. Paterson, H. Kamencic, A. Shoker, A.W. Lyon, Pre-column derivatization high-performance liquid chromatographic method for determination of cysteine, cysteinyl-glycine, homocysteine and glutathione in plasma and cell extracts, Journal of Chromatography B: Biomedical Sciences and Applications 758 (2001) 20712. 
[16] G. Chwatko, E. Bald, Determination of cysteine in human plasma by highperformance liquid chromatography and ultraviolet detection after pre-column derivatization with 2-chloro-1-methylpyridinium iodide, Talanta 52 (2000) 509-15.

[17] X. Guan, B. Hoffman, C. Dwivedi, D.P. Matthees, A simultaneous liquid chromatography/mass spectrometric assay of glutathione, cysteine, homocysteine and their disulfides in biological samples, Journal of Pharmaceutical and Biomedical Analysis 31 (2003) 251-61.

[18] D. Tsikas, M. Raida, J. Sandmann, S. Rossa, W.-G. Forssmann, J.C. Frölich, Electrospray ionization mass spectrometry of low-molecular-mass S-nitroso compounds and their thiols, Journal of Chromatography B: Biomedical Sciences and Applications 742 (2000) 99-108.

[19] T. Inoue, J.R. Kirchhoff, Determination of Thiols by Capillary Electrophoresis with Amperometric Detection at a Coenzyme Pyrroloquinoline Quinone Modified Electrode, Analytical Chemistry 74 (2002) 1349-54.

[20] W. Jin, Y. Wang, Determination of cysteine by capillary zone electrophoresis with end-column amperometric detection at a gold/mercury amalgam microelectrode without deoxygenation, Journal of Chromatography A 769 (1997) 307-14.

[21] J.V. Ros-Lis, B. García, D. Jiménez, R. Martínez-Máñez, F. Sancenón, J. Soto, F. Gonzalvo, M.C. Valldecabres, Squaraines as Fluoro-Chromogenic Probes for ThiolContaining Compounds and Their Application to the Detection of Biorelevant Thiols, Journal of the American Chemical Society 126 (2004) 4064-5.

[22] J. Du, Y. Li, J. Lu, Investigation on the chemiluminescence reaction of luminolH2O2-S2-/R-SH system, Analytica Chimica Acta 448 (2001) 79-83.

[23] A. Brotons, I. Sanjuan, C.E. Banks, F.J. Vidal-Iglesias, J. Solla-Gullón, J. Iniesta, Voltammetric behaviour of 7-methylguanine using screen-printed graphite electrodes: towards a guanine methylation electrochemical sensor, Electroanalysis (2015) n/a-n/a.

[24] I. Sanjuan, A. Brotons, N. Hernandez-Ibanez, C.W. Foster, C.E. Banks, J. Iniesta, Boron-doped diamond electrodes explored for the electroanalytical detection of 7methylguanine and applied for its sensing within urine samples, Electrochim. Acta 197 (2016) 167-78.

[25] J.A. Reynaud, B. Malfoy, P. Canesson, Electrochemical investigations of amino acids at solid electrodes: Part I. Sulfur components: Cystine, cysteine, methionine, Journal of Electroanalytical Chemistry and Interfacial Electrochemistry 114 (1980) 195211.

[26] M.E. Johll, D.G. Williams, D.C. Johnson, Activated pulsed amperometric detection of cysteine at platinum electrodes in acidic media, Electroanalysis 9 (1997) 1397-402.

[27] L. Zhang, L. Ning, Z. Zhang, S. Li, H. Yan, H. Pang, H. Ma, Fabrication and electrochemical determination of 1-cysteine of a composite film based on V-substituted polyoxometalates and $\mathrm{Au} @ 2 \mathrm{Ag}$ core-shell nanoparticles, Sensors and Actuators B: Chemical 221 (2015) 28-36.

[28] R. Domínguez, B. Serra, A.J. Reviejo, J.M. Pingarrón, Chiral Analysis of Amino Acids Using Electrochemical Composite Bienzyme Biosensors, Analytical Biochemistry 298 (2001) 275-82.

[29] R.P. Deo, N.S. Lawrence, J. Wang, Electrochemical detection of amino acids at carbon nanotube and nickel-carbon nanotube modified electrodes, Analyst 129 (2004) 1076-81.

[30] M.W. Hsiao, R.R. Adzic, E.B. Yeager, Electrochemical oxidation of glucose on single crystal and polycrystal line gold surfaces in phosphate buffer, Journal of the Electrochemical Society 143 (1996) 759-67. 
[31] L.Z. Pei, Z.Y. Cai, Y.Q. Pei, Y.K. Xie, C.G. Fan, D.G. Fu, Electrochemical determination of L-cysteine using polyaniline/CuGeO3 nanowire modified electrode, Russ. J. Electrochem. 50 (2014) 458-67.

[32] H. Xu, J. Xiao, B. Liu, S. Griveau, F. Bedioui, Enhanced electrochemical sensing of thiols based on cobalt phthalocyanine immobilized on nitrogen-doped graphene, Biosensors and Bioelectronics 66 (2015) 438-44.

[33] C.C. Corrêa, S.A.V. Jannuzzi, M. Santhiago, R.A. Timm, A.L.B. Formiga, L.T. Kubota, Modified electrode using multi-walled carbon nanotubes and a metallopolymer for amperometric detection of 1-cysteine, Electrochimica Acta 113 (2013) 332-9.

[34] P. Swetha, A.S. Kumar, Phosphomolybdic acid nano-aggregates immobilized nafion membrane modified electrode for selective cysteine electrocatalytic oxidation and anti-dermatophytic activity, Electrochimica Acta 98 (2013) 54-65.

[35] M. Ahmad, C. Pan, J. Zhu, Electrochemical determination of 1-Cysteine by an elbow shaped, Sb-doped $\mathrm{ZnO}$ nanowire-modified electrode, Journal of Materials Chemistry 20 (2010) 7169-74.

[36] M. Rangel Argote, E. Sánchez Guillén, A.G. Gutiérrez Porras, O. Serrano Torres, C. Richard, J.H. Zagal, F. Bedioui, S. Gutierrez Granados, S. Griveau, Preparation and Characterization of Electrodes Modified with Pyrrole Surfactant, Multiwalled Carbon Nanotubes and Metallophthalocyanines for the Electrochemical Detection of Thiols, Electroanalysis 26 (2014) 507-12.

[37] J.H. Zagal, P. Herrera, Electrochemistry of cysteineand cystine on metalphthalocyaninesadsorbed on a graphite electrode, Electrochim. Acta 30 (1985) 449-54.

[38] M.K. Halbert, R.P. Baldwin, Electrocatalytic and analytical response of cobaltphthalocyanine containing carbon paste electrodestoward sulfhydryl compounds, Anal. Chem. 57 (1985) 591-5.

[39] K.I. Ozoemena, J. Pillay, T. Nyokong, Preferential electrosorption of cobalt (II) tetra-aminophthalocyanine at single-wall carbon nanotubes immobilized on a basal plane pyrolytic graphite electrode, Electrochemistry Communications 8 (2006) 1391-6.

[40] R.d.C.S. Luz, A.B. Moreira, F.S. Damos, A.A. Tanaka, L.T. Kubota, Cobalt tetrasulphonated phthalocyanine immobilized on poly-l-lysine film onto glassy carbon electrode as amperometric sensor for cysteine, Journal of Pharmaceutical and Biomedical Analysis 42 (2006) 184-91.

[41] I.S. da Silva, M.F. Antao Araujo, H.A. Ferreira, J.d.J. Gomes Varela, Jr., S.M. Carvalho Neiva Tanaka, A.A. Tanaka, L. Angnes, Quantification of N-acetylcysteine in pharmaceuticals using cobalt phthalocyanine modified graphite electrodes, Talanta 83 (2011) 1701-6.

[42] C. Foster, J. Pillay, J. Metters, C. Banks, Cobalt Phthalocyanine Modified Electrodes Utilised in Electroanalysis: Nano-Structured Modified Electrodes vs. Bulk Modified Screen-Printed Electrodes, Sensors 14 (2014) 21905.

[43] K. Honeychurch, L. Gilbert, J. Hart, Electrocatalytic behaviour of citric acid at a cobalt phthalocyanine-modified screen-printed carbon electrode and its application in pharmaceutical and food analysis, Anal Bioanal Chem 396 (2010) 3103-11.

[44] C.W. Foster, J.P. Metters, D.K. Kampouris, C.E. Banks, Ultraflexible ScreenPrinted Graphitic Electroanalytical Sensing Platforms, Electroanalysis 26 (2014) 26274.

[45] C.-Y. Liao, J.-M. Zen, Development of a method for total plasma thiols measurement using a disposable screen-printed carbon electrode coupled with a $\mathrm{MnO} 2$ reactor, Sensors and Actuators B: Chemical 129 (2008) 896-902. 
[46] W.-Y. Su, S.-H. Cheng, Electrocatalysis and sensitive determination of cysteine at poly(3,4-ethylenedioxythiophene)-modified screen-printed electrodes, Electrochemistry Communications 10 (2008) 899-902.

[47] M. Vasjari, A. Merkoçi, P.J. Hart, S. Alegret, Amino Acid Determination Using Screen-Printed Electrochemical Sensors, Microchimica Acta 150 (2005) 233-8.

[48] A.J. Bard, L.R. Faulkner, Electrochemical Methods: Fundamentals and Applications, Wiley, 2000.

[49] B. Filanovsky, Electrochemical response of new carbon electrodes bulk modified with cobalt phthalocyanine to some thiols in the presence of heptane or human urine, Analytica Chimica Acta 394 (1999) 91-100.

[50] Q. Li, C. Batchelor-McAuley, R.G. Compton, Electrochemical Oxidation of Guanine: Electrode Reaction Mechanism and Tailoring Carbon Electrode Surfaces To Switch between Adsorptive and Diffusional Responses, The Journal of Physical Chemistry B 114 (2010) 7423-8.

[51] N. Spãtaru, B.V. Sarada, E. Popa, D.A. Tryk, A. Fujishima, Voltammetric Determination of 1-Cysteine at Conductive Diamond Electrodes, Analytical Chemistry 73 (2001) 514-9.

[52] M.A. Gulppi, M.A. Paez, J.A. Costamagna, G. Cardenas-Jiron, F. Bedioui, J.H. Zagal, Inverted correlations between rate constants and redox potential of the catalyst for the electrooxidation of 2-aminoethanethiol mediated by surface confined substituted cobalt-phthalocyanines, J. Electroanal. Chem. 580 (2005) 50-6.

[53] S. Griveau, V. Albin, T. Pauporte, J.H. Zagal, F. Bedioui, Comparative study of electropolymerized cobalt porphyrin and phthalocyanine based films for the electrochemical activation of thiols, J. Mater. Chem. 12 (2002) 225-32.

[54] J.H. Zagal, S. Griveau, J. Francisco Silva, T. Nyokong, F. Bedioui, Metallophthalocyanine-based molecular materials as catalysts for electrochemical reactions, Coord. Chem. Rev. 254 (2010) 2755-91.

[55] M. Zhou, J. Ding, L.-p. Guo, Q.-k. Shang, Electrochemical Behavior of 1-Cysteine and Its Detection at Ordered Mesoporous Carbon-Modified Glassy Carbon Electrode, Analytical Chemistry 79 (2007) 5328-35.

[56] A. Salimi, S. Pourbeyram, Renewable sol-gel carbon ceramic electrodes modified with a Ru-complex for the amperometric detection of 1-cysteine and glutathione, Talanta 60 (2003) 205-14.

[57] A. Brotons, I. Sanjuan, C.E. Banks, F. Jose Vidal-Iglesias, J. Solla-Gullon, J. Iniesta, Voltammetric Behaviour of 7-Methylguanine Using Screen-printed Graphite Electrodes: towards a Guanine Methylation Electrochemical Sensor, Electroanalysis 27 (2015) 2766-72.

[58] D.E. Morbeck, R.L. Krisher, J.R. Herrick, N.A. Baumann, D. Matern, T. Moyer, Composition of commercial media used for human embryo culture, Fertility and Sterility 102 (2014) 759-U471. 